\title{
Chilled Cabbage Leaves: The Possible Remedy for Breast Engorgement
}

\author{
Rajashree Salgaonkar \\ Department of Obstetrics and Gynecological Nursing, College of Nursing, Bharati Vidyapeeth, Navi Mumbai, Maharashtra, India
}

\section{Abstract}

Breast engorgement is defined as congestion and distension with fluid. The lactation literature defines it as a swollen breast, caused by the buildup of breast milk during breastfeeding, and is often referred to as swelling and distension of the breasts and can be a painful condition. This is a common physiological problem for lactating mothers that are caused by the abrupt boost in the volume of the breast milk due to lymphatic and vascular congestion with interstitial edema during the first 2 weeks of breastfeeding. The rationale why engorgement occurs in the first 2 weeks postpartum is that the mother and her baby are adjusting to the process of demand and supply, i.e., if the milk production is inflated too speedily, this might exceed the capability of the breast alveoli to store it. Breast engorgement may occur due to scarce emptying of the breast milk from the mother due to poor transfer of breast milk and incorrect latching or positioning of the baby during the course of suckling. Engorgement of the breasts is associated with severe pain, nipple tenderness, fissures of the nipple, and infection which can all result in subsequent cessation of breastfeeding. Many methods for the treatment of breast engorgement have been explored. Some of the non-medical interventions include cold cabbage compresses cold gel pads, hot compresses, and warm showers, which are used to activate the milk ejection reflex besides the use of medical treatments such as the use of diuretics. Thus, the present review focuses on the use of chilled cabbage leaves for the treatment of breast engorgement.

Keywords: Cabbage leaves, breast engorgement, breast milk

\section{INTRODUCTION}

"Mother, the most beautiful on the lips of mankind" ---Kahilil Gibran.

Motherhood is the only act that manifested in human form the cosmic wonder of creation. Childbirth is a process beautifully designed by nature and the care following the birth of the baby also essential for the maintenance of the health of both mother and child. ${ }^{[1]}$ Childbirth is a transcendent even with meaning far beyond the actual physiologic process. The main vital consideration for the infant in tropical countries is breastfeeding and avoidance of infection. Breast milk is

\begin{tabular}{|l|}
\hline \multicolumn{1}{|c|}{ Access this article online } \\
\hline Website: http://innovationalpublishers.com/Journal/ijnmi \\
\hline ISSN No: $2656-4656$ \\
DOI: $10.31690 /$ ijnmi/30 \\
\hline
\end{tabular}

the food of choice for infants. Breastfeeding offers many advantages, nutritional, immunological, and psychological. ${ }^{[2]}$

The primi mother and the mother within elastic breast are likely to be involved in developing breast complication. The factors that are exaggerated normal venous and lymphatic enlargement of breast which precedes lactation. This, in turn, prevents escape of milk from the lacteal system. The nipple may become pain due to loss of surface epithelium with the formation of a row area on the nipple, due to a fissure situated either at tip of the base or of the nipple. ${ }^{[3]}$

The common breast complication are breast engorgement, cracked and retracted nipples, leading to difficulty in breastfeeding mastitis, breast abscess, and lactation failure. Breast engorgement and infection are responsible for puerperal pyrexia. ${ }^{[4]}$ Breast fullness is a normal part of lactation which nearly all women experience when their milk comes in 2-5 days after birth. This feeling of fullness, which may be accompanied by a feeling of heaviness, tenderness, and

Address for Correspondence:

Ms. Rajashree Salgaonkar, Department of Obstetrics and Gynecological Nursing, College of Nursing, Bharti Vidyapeeth, Navi Mumbai, Maharashtra, India. E-mail: salgaonkarrajshri@gmail.com

This is an open-access journal, and articles are distributed under the terms of the Creative Commons Attribution Noncommercial Share Alike 4.0 License, which allows others to remix, tweak, and build upon the work non-commercially, as long as appropriate credit is given and the new creations are licensed under the identical terms 
warmth, is caused by swelling of the breast tissue such as blood, lymphatic fluid, and milk collecting duct as the process of milk production begins with this normal fullness, the breast tissue is compressible and generally feel well.

\section{Breast engorgement}

It is the most common complication during the postnatal period. It is the disease condition occurring in the mammary glands by expanding veins and the pressure of new breast milk contained within them. The most common manifestation includes considerable pain and feeling of tenderness in the breast, generalized malaise, rise in temperature, and painful breastfeeding. Severe engorgement leads to mastitis and untreated engorgement puts pressure on the milk duct often causing the plugged nipple. ${ }^{[5,6]}$

\section{Preventing engorgement}

- The preventive aspect of breast engorgement is feed frequently. The mother has to nurse at least 10-12 times in $24 \mathrm{~h}$ during the day with no $>3 \mathrm{~h}$ stretch at night. Try to nurse at least $15 \mathrm{~min}$ on the first side before offering the second.

- Do not use a pacifier since you might miss your baby's feeding cues.

- Do not time or limit feeds. Allow baby to end the feed himself when he is done.

- Applying ice to breasts, as "milk comes in" (after feeds for 15-20 min) will reduce the swelling caused by edema. A bag of frozen peas or corn works well.

\section{Managing engorgement Cold compress}

Application of cold compress to the breasts and under the arms between feedings helps to reduce swelling. It should be for 15-20 min and on for 1-2 h. Women have been using cabbage leaves to relieve breast engorgement symptoms for several years. It is a natural remedy which really works on breast engorgement. ${ }^{[7]}$

\section{Hot water application}

The other treatments include hot water application, gentle breast massage, manual expression, or pump a little milk from the breast, administer analgesics of pain.

Engorgement can be defined as congestion, distention with fluid literature refers to engorgement as the physiologic condition characterized by the painful swelling of the breasts associated with the sudden increase in milk volume, lymphatic and vascular congestion, and interstitial edema during the first 2 weeks following birth engorgement is a normal physiologic process with a progression of events, not a result of trauma or injury to tissues (American Journal of Obstetrics and Gynecology, 2007).

\section{Cabbage leaves}

The application of cabbage leaves to the mother's breasts between breastfeedings to reduce engorgement is another strategy that has been used by some breastfeeding women, but for which the evidence of effectiveness is inconclusive due to methodological limitations. The Cochrane review found no statistically significant evidence that the use of cabbage leaves was associated with a more rapid resolution of engorgement symptoms. ${ }^{[7]}$

Despite this, many women find the application of cabbage leaves to be soothing. It is not necessary to chill cabbage leaves (Roberts et al., 1995). Mothers should be informed that their breasts might smell or taste-like cooked cabbage.

These non-medical interventions are receiving increasing attention as feasible treatment methods as they are more easily available and generally easy to use, convenient, and cheap as compared to medical interventions. ${ }^{[8,9]}$

Cabbage leaves contain natural mixture of ingredients such as sinigrin rapine, mustard oil, magnesium, oxalate, and sulfur heterosides which help to decrease tissue congestion by dilatory local capillaries in mild, moderate, and severe discomfort. Cabbage has both anti-inflammatory and antiirritant properties. ${ }^{[10]}$

\section{Need of the study}

"Breast milk is not only the best but must for the infants" --- WHO, 2005.

The most often stated reason for cessation of breastfeeding in the first 2 weeks of postpartum is pain. Breast engorgement is a painful problem that can lead to premature weaning. It is a common complication of the early puerperium and usually occurs between 3 and 6 days after delivery. Dr. Ruth Lawrence defines engorgement as "the swelling and distention of breasts usually in the early days of initiation of lactation, due to vascular dilatation as well as early arrival of milk." Immediately after birth, the mother produces colostrum, a yellow sticky fluid secreted by breasts provides nutrition and protects against infection. ${ }^{[11]}$

Breast engorgement usually happens when the breast switch off from colostrum to mature milk. It can also happen if lactating women miss several nursing and not enough milk is expressed from breasts.

Breast engorgement is a painful, unpleasant condition affecting large number of women in early postpartum. During a time, when mothers are coping with demands of the baby, it may be particularly distressing breast engorgement inhibits the development of successful breastfeeding, leading to early breastfeeding cessation, an associated with more serious illness including breast infection.

Numerous strategies have been adopted over the years in the treatment of breast engorgement, but very few researches have been conducted to monitor the effect of cabbage leaves on breast engorgement.

Cabbage leaves have been used for centuries as a folk remedy for a wide variety of ailments and received much-renewed interest from lactation professionals over the past 10 years. 
It contains natural mixture of ingredients which helps to decrease tissue congestion by dilatory local capillaries in mild, moderate, and severe discomfort. Hot application reduces pain and causes relaxation of blood vessels, thereby opening vessels and increasing blood flow.

A study has conducted a quasi-experimental study in an effect of chilled cabbage leaves on the treatment of breast engorgement in Thailand. The experimental group received chilled cabbage leaf application and the controlled group received routine care. This study concluded that chilled cabbage leaf application had great improvement in treating this condition. ${ }^{[12]}$

A quasi-experimental study was conducted in Tamil Nadu to assess the effectiveness of cabbage leaves application on breast engorgement among postnatal mothers. The study comprises 24 mothers selected using purposive sampling method. Pretreatment level of breast engorgement compared with posttreatment level and found that application of cabbage leaves to relieve breast engorgement is very effective (Bhuvaneswari, 2010).

A study was conducted in Australia to compare the effectiveness of chilled and room temperature green cabbage leaves in reducing discomfort of breast engorgement among postnatal mothers. 28 lactating women with breast engorgement used chilled cabbage leaves or on breast and room temperature cabbage leaves on other breasts for $2 \mathrm{~h}$ period. Pre-treatment pain level was compared with post-treatment levels for both the conditions. Mothers reported less pain with both the conditions and majority of mothers preferred cold cabbage leaves (Mrs. Diane Margaret, 2002).

\section{Conclusion}

Breast engorgement is one of the most common minor discomforts of the women after delivery, especially primipara. Breast engorgement is a physiological condition that is characterized by painful, swelling of the breast as a result of a sudden increase in milk volume, lymphatic and vascular congestion, and interstitial edema during the first 2 weeks following childbirth, this condition is caused by insufficient breastfeeding or obstruction in milk ducts. Breast pain during breastfeeding is a common problem that interferes with successful breastfeeding, leading to engorgement. ${ }^{[13]}$ Thus, the present study was undertaken to find out the effect of cabbage leaves on relief breast engorgement among postnatal women.

\section{RefEREnCes}

1. Chopra M. Breast Milk is Best but What About HIV. MRC News; 2006. Available from: http://www. mrc.ac.za/mrcnews/dec2006/breast.htm. [Last accessed on 2006 Dec 12]

2. Padubidri V, Anand E, editors. A Text Book of Obstetrics. $1^{\text {st }}$ ed. New Delhi: BL Publications; 2006. p. 390-1.

3. Dutta DC, Konar H. Textbook of Obstetrics Including Perinatology and Contraception. $6^{\text {th }}$ ed. Calcutta: New Central Book Agency (P) Ltd.; 2004. p. 149-50, 451-3.

4. Giugliani ER. Common problems during lactation and their management. J Pediatr (Rio J) 2004;80:S147-54.

5. Lawrence RL. Breastfeeding: A Guide for the Medical Professions. St Louis: Mosby; 2005.

6. Lawrence R, Lawrence RM. Breastfeeding: A Guide for the Medical Professions. $6^{\text {th }}$ ed. St Louis: Mosby; 2005.

7. Mangesi L, Dowswell T. Treatments for breast engorgement during lactation. Cochrane Database Syst Rev 2010;9:CD006946.

8. Arora S, Vatsa M, Dadhwal V. A comparison of cabbage leaves vs. Hot and cold compresses in the treatment of breast engorgement. Indian J Community Med 2008;33:160-2.

9. Robson BA. Breast Engorgement in Breastfeeding Mothers. Cleveland: Case Western Reserve University (health sciences); 1990.

10. Roberts KL. A comparison of chilled cabbage leaves and chilled gelpaks in reducing breast engorgement. J Hum Lact 1995;11:17-20.

11. Campbell S, Lees C. Obstetrics by 10 teachers. $17^{\text {th }}$ ed. London: Arnold; PN 87-100, Sep 20, 2000.

12. Ruba R. Effectiveness of cabbage leaves application to relieve breast engorgement. Nightingale Nurs Times 2009;5:48-51.

13. Kathryn LR, Reiter M, Schuster D. Effects of cabbage leaf extract on breast engorgement. J Hum Lact 1998;14:231-6.

How to cite this article: Salgaonkar R, Chilled Cabbage Leaves: The Possible Remedy for Breast Engorgement. Int J Nurs Med Invest. 2019;4(1):1-3 